Daniel Kremers* und Shunsuke Izuta

\title{
Bedeutungswandel der Zivilgesellschaft oder das Elend der Ideengeschichte: Eine kommentierte Übersetzung von Hirata Kiyoakis Aufsatz zum Begriff shimin shakai bei Antonio Gramsci (Teil 1)
}

\author{
Kiyoaki Hirata: Über Gramscis Begriff der bürgerlichen \\ Gesellschaft (Teil 1: Hegel und Marx im Begriff der bürgerlichen \\ Gesellschaft)
}

DOI 10.1515/asia-2017-0044

\begin{abstract}
The history of ideas is a history of translations and interpretations, of finding new words for old phenomena and attributing new phenomena to old words. In this commented translation from a Japanese source text, this historical process is demonstrated for the term civil society and the languages German, French, Italian and Japanese. In his 1989 article "On Gramsci's notion of civil society", Japanese Marxist Kiyoaki Hirata compared the use of the term by Georg W. F. Hegel, Karl Marx and Antonio Gramsci, while translating it to Japanese as shimin shakai 市民社会, today a highly popular term in Japan. We have

Anmerkungen zu Übersetzung und Zitationsweise: Namen werden mit dem Vornamen voran wiedergegeben. Die Umschrift japanischer Begriffe in lateinischen Lettern (romaji) erfolgt nach dem Hepburn-System. Von den Übersetzern hinzugefügte Umschriften sowie Übersetzungen von Titeln im Original erwähnter Publikationen sind durch eckige Klammern gekennzeichnet. Runde Klammern außerhalb von eckigen Klammern entsprechen der Setzung des Autors im Original. Alle anderen typografischen Sonderzeichen innerhalb der Übersetzung entsprechen Hervorhebungen des Autors im Original. Anmerkungen und Kommentare der Übersetzer befinden sich in den Fußnoten.
\end{abstract}

Originaltitel: Guramushi no shimin shakai gainen ni yosete (I: Shimin shakai gainen ni okeru Hehgeru to Marukusu) グラムシの市民社会概念によせて (I: 市民社会概念におけるへーゲルと マルクス), von Kiyoaki Hirata 平田清明

*Corresponding author: Daniel Kremers, Deutsches Institut für Japanstudien (DIJ), Tokyo, Jochi Kioizaka Bldg. 2F, 7-1 Kioicho, Chiyoda-ku, Tokyo 102-0094, Japan. E-mail: kremers@dijtokyo.org Shunsuke Izuta, Faculty of Foreign Languages, Department of German, Dokkyo University, 1-1 Gakuen-cho, Soka-shi, Saitama 340-0042, Japan. E-mail: izuta@dokkyo.ac.jp 
translated the first part of Hirata's article, in which he endeavors on the connections and differences between Hegel and Marx. His major finding is that Karl Marx in Misère de la philosophie (1847) has taken Hegel's concept of civil society or civic community (bürgerliche Gesellschaft), splitting it in two and so differentiating between civil society (société civile) and bourgeois society (société bourgeoise). However, as Hirata noticed, when Misère de la philosophie was translated to German as Das Elend der Philosophie in 1888 this differentiation was lost. The German version, just like Hegel's Grundlinien der Philosophie des Rechts only spoke of bürgerliche Gesellschaft. When Hirata translated the text to Japanese, however, he opted for using the French original as source text, thus distinguishing between shimin shakai and burujoa shakai. What Hirata does not mention though, is that by translating the French société bourgeoise to German as bürgerliche Gesellschaft, Eduard Bernstein, Karl Kautsky and Friedrich Engels have cut off the German discussion on bürgerliche Gesellschaft from the global discussion of civil society for more than a century. Only in the late 1980s, German speaking Marxist intellectuals engaging with the works of Antonio Gramsci began to realize that there is more to bürgerliche Gesellschaft than being a translation for société bourgeoise. This inspired them even to invent the new word Zivilgesellschaft as a translation for the Gramscian società civile a linguistic trick that is peculiar to the German language, and became necessary because the German language had lacked a marker for the difference between bourgeois and citoyen. In the following years however, Zivilgesellschaft became such a popular term that has by now replaced Hegel's bürgerliche Gesellschaft as a translation for civil society in many contexts and has been attributed many contested meanings - way beyond the realm of Marxist theory. These processes of translations and transformation are usually invisible in our daily use of language, and become only apparent when we actively compare and interpret sources from different epochs and languages.

Keywords: history of ideas, civil society, Hegel, Marx, Japan

Die europäische politische Philosophie, die zu der des Westens geworden ist und sich noch immer weiter über dessen Grenzen vorschiebt, lässt sich als eine Folge von Übersetzungen beschreiben, und zwar im Doppelsinn: Äquivalenzbildung in einer anderen Sprache und Übertragung auf einen anderen Sachverhalt, der durch einen historischen Einschnitt vom ursprünglich so Bezeichneten geschieden ist.

Wolfgang Fritz Haug (1994: 494) 


\section{Einleitung}

Die Wiederentdeckung des Begriffs der civil society (shimin shakai 市民社会) in der Politik und den Sozialwissenschaften ab Ende der 1980er Jahre - im Deutschen begleitet von einer Neuschöpfung (Zivilgesellschaft) und Umdeutungen (Bürgergesellschaft) - wurde ausgelöst durch den Versuch der sozialistischen Machthaber in Polen, den aufkeimenden sozialen Bewegungen und Protesten ein marxistisch klingendes Etikett anzuhängen. ${ }^{1}$ Die Wahl fiel auf „bürgerliche Gesellschaft” (civil society / spoleczenstwo obywatelskie). ${ }^{2}$ Diese Ereignisse fielen zeitlich zusammen mit dem fünfzigsten Todestag des italienischen Kommunisten Antonio Gramsci (1891-1937) sowie dem Beginn der Übersetzung seiner Gefängnishefte ins Englische und Deutsche. ${ }^{3}$

Auch in Japan wurde Gramscis Hauptwerk ab Mitte der 1980er Jahre stärker wahrgenommen. 1989 erschien bei Shakai Hyōronsha ein Sammelband mit dem Titel Lebender Gramsci. ${ }^{4}$ Der von Kiyotomo Ishidō, Momo Iida und Kaoru Katagiri herausgegeben Band enthielt auf 550 Seiten 36 wissenschaftliche Beiträge, die sich mit Gramscis Leben und Denken sowie mit deren Bedeutung für Kultur und soziale Bewegungen in Japan auseinandersetzten. Darüber hinaus enthielt der Band mehrere kommentierte Übersetzungen von an Gramsci gerichteten Briefen, eine Bibliographie japanischsprachiger Veröffentlichungen über Gramsci sowie eine Kurzbiografie.

Ein zentrales Element der Gesellschaftstheorie Gramscis ist sein Begriff der bürgerlichen Gesellschaft bzw. Zivilgesellschaft (società civile). Dieser widmete sich innerhalb jenes Sammelbandes ein Beitrag von Hirata Kiyoaki (1922-1995) ${ }^{5}$

1 Buttigieg 1991: 9; Dahrendorf 1992: 35; Demirović 1991: 41; Habermas 1992: 399; Haug 1988: 38, 1989: 72; 1994: 494; Reiman 1987: 21.

2 Zaleski 2007: 222. Pawel Zaleski räumt auf mit dem Mythos, dass bürgerliche Gesellschaft ein zentraler Begriff osteuropäischer Oppositioneller und Dissidenten gewesen sei, vgl. Zaleski 2007. Dieser tauchte erst 1987 in oppositionellen Schriften auf. Vermehrt gebraucht wurde er 1989 durch die sozialistischen Machthaber innerhalb der Verhandlungen am "runden Tisch". Mit dem Rückgriff auf den marxistischen Begriff versuchte der Staatssozialismus einen Raum für freie Zusammenschlüsse zu benennen ohne das politische System zu reformieren. Der Begriff wurde in der Folge durch die westliche Berichterstattung über diese Ereignisse popularisiert.

3 Gramsci et al. 1992-2007; Gramsci et al. 1991-2002.

4 Ishido et al. 1989.

5 Der japanische Wirtschaftshistoriker und -theoretiker Kiyoaki Hirata hat die marxistische Theorie im Japan der Nachkriegszeit als Vertreter der sogenannten „Theorie der bürgerlichen Gesellschaft“ (shimin shakairon 市民社会論) wesentlich mitgeprägt. In Abgrenzung zum staatssozialistischen Marxismus-Leninismus bemühte sich Hirata um die Nutzbarmachung des Begriffs der bürgerlichen Gesellschaft als Kategorie einer materialistischen Gesellschaftstheorie. Anders als der andere bekannte Vertreter der shimin shakairon, Yoshihiko Uchida (1913-1989), ging es Hirata weniger um eine Unterscheidung zwischen bürgerlichen (England) und feudalistischen Gesellschaften 
mit dem vielversprechenden Titel „Über Gramscis Begriff der bürgerlichen Gesellschaft“ . ${ }^{6}$ Bei einer genaueren Betrachtung des Textes stellte sich jedoch heraus, dass Hirata, der vielen als Vertreter der „Theorie der bürgerlichen Gesellschaft“ (shimin shakairon) innerhalb des japanischen Marxismus bekannt ist, sich weniger für Gramsci und mehr für den Begriff der bürgerlichen Gesellschaft bei Marx interessierte. ${ }^{7}$

So handelt es sich bei seinem Beitrag weniger um eine intensive Auseinandersetzung mit den Schriften des unter dem italienischen Faschismus inhaftierten Kommunisten, als um eine Gegenüberstellung seiner Begrifflichkeiten mit denen Marx' und Hegels. Dies wird besonders deutlich darin, dass es sich bei Hiratas Beitrag nicht um ein originäres Werk handelt, sondern um eine - wenn auch erheblich erweiterte - Neufassung eines bereits 1987 in der Vierteljahreszeitschrift Kikan Kuraishisu erschienen achtseitigen Essays mit dem Titel „Marx’ Begriff der bürgerlichen Gesellschaft überdenken“. ${ }^{8}$ Dessen erweiterte Fassung von 1989 besteht damit aus zwei Teilen, erstens dem bereits erschienen Essay zum Begriff der bürgerlichen Gesellschaft bei Hegel und Marx sowie zweitens einem Aufsatz über die Adaption des Begriffs durch Antonio Gramsci. Wir legen hier eine Übersetzung des ersten Teils in der Fassung von 1989 vor. ${ }^{9}$

Hiratas primäre Quellen in diesem sind Hegels Grundlinien der Philosophie des Rechts (GPR), darin vor allem der dritte Abschnitt „Der Staat“ des dritten Teils „Die Sittlichkeit“, und Marx’ Misère de la philosophie sowie dessen deutsche Übersetzung Das Elend der Philosophie. Dabei machte Hirata eine interessante „Entdeckung“, nämlich, dass Marx in der französischen Fassung von

(Japan), sondern um eine Weiterentwicklung marxistischer Begrifflichkeiten für ein besseres Verständnis des Gegenwartskapitalismus, vgl. Avenell 2011: 324. Sein Ausgangspunkt dabei war die Unterscheidung zwischen société civile und société bourgeoise in Marx' Misère de la philosophie, welche er 1948 ins Japanische übersetzt hatte. In den 1980er Jahren setzte er sich intensiv mit der französischen Schule der Regulationstheorie auseinander, vgl. Hirata 1989: 137; Seifert 1999: 23; sowie Liepitz 1992. Hirata wurde als Nachfahre einer Adelsfamilie aus Satsuma (heute Präfektur Kagoshima) 1922 in Tokyo geboren. 1949 schloss er sein Studium an der Handelshochschule Tokio (heute Hitotsubashi University) ab. 1970 wurde er Professor am wirtschaftswissenschaftlichen Institut der Universität Nagoya. 1978 folgte er einem Ruf an das wirtschaftswissenschaftliche Institut der Universität Kyoto. Von 1984 bis 1985 war er Gastprofessor an der Universität von Paris, Sorbonne. 1988 erhielt er eine Ehrenprofessur an der Universität Kyoto. Er starb 1995 in Kagoshima, Hishiyama 1995; Yagi 1995.

6 Hirata 1989.

7 Foljanty-Jost/Haufe 2006: 249; Kato 1991: 16; Seifert 1999: 23; Tsujinaka 2010; Yagi 2005.

8 Hirata 1987a.

9 Die Idee zu dieser Übersetzung entstand während unserer Zeit am Internationalen Graduiertenkolleg „Formwandel der Bürgergesellschaft - Japan und Deutschland im Vergleich“ (IGK) HalleTokyo. 
Misère de la philosophie zwischen société civile und société bourgeoise unterschied, in der deutschen Übersetzung (die Hirata ebenfalls Marx zuschreibt) aber meist nur von bürgerlicher Gesellschaft die Rede ist. ${ }^{10}$ Damit ist Hirata, ohne es $\mathrm{zu}$ bemerken, auf den Ursprung eines Bruchs in der deutschsprachigen Ideenund Begriffsgeschichte der bürgerlichen Gesellschaft gestoßen, der trotz intensiver Diskussion im Deutschen so noch nicht benannt wurde. Die Relevanz von Hiratas „Entdeckung“ für die Begriffsgeschichte sei im Folgenden dargestellt.

\subsection{Bürgerliche Gesellschaft übersetzen}

Die Wiederentdeckung der civil society ab Ende der 1980er Jahre ging im Deutschen mit der Erfindung des Wortes Zivilgesellschaft einerseits und der Umdeutung des Ausdrucks Bürgergesellschaft andererseits einher. „Zivilgesellschaft“ wurde von Wolfgang Fritz Haug gewählt, um einerseits Gramscis società civile und andererseits das von Gorbatschow aufgegriffene grazhdanskoye obshchestvo (гражданское общество) ins Deutsche zu übersetzen. ${ }^{11} 1992$ deutete Jürgen Habermas den Begriff um. In Faktizität und Geltung markiert die Zivilgesellschaft einen Teilbereich des Habermasschen Öffentlichkeitsbegriffs - den der freiwilligen Vereinigungen, des gewaltlosen Protestes und zivilen Ungehorsams. ${ }^{12}$ In ähnlicher Weise wurde der Begriff „Bürgergesellschaft“, der lange davor gleichbedeutend mit Verein war, von Michael Reiman benutzt, um grazhdanskoye obshchestvo zu übersetzen. ${ }^{13}$ Für Ralf Dahrendorf erschien der Begriff als geeignete Übersetzung für das englische civil society. ${ }^{14}$ Grund für diese Wortspielereien war, dass das deutsche Adjektiv bürgerlich als Entsprechung sowohl für das französische civile als auch bourgeoise herhalten muss, und damit für das nun $\mathrm{zu}$ Bezeichnende ungeeignet erschien.

Der begriffsgeschichtliche Ursprung dieses Dilemmas liegt in Karl Marx' Misère de la philosophie von 1847, in dem dieser eine Unterscheidung von société civile und société bourgeoise vorgenommen hatte. ${ }^{15}$ In der französischen Originalfassung erscheint société civile als ein die Epochen übergreifender sowie Überbau und Basis vereinender Oberbegriff - analog $\mathrm{zu}$ dem Begriff der

10 Hirata 1989: 122.

11 Haug 1988, 1989.

12 Habermas 1992: 464.

13 Lange 1961; Reiman 1987: 21.

14 Dahrendorf 1992: 44; vgl. Hettling/Foljanty-Jost 2009: 21-21.

15 Hirata 1989: 122; Marx 1968 [1847]. 
„Sittlichkeit“ bei Hegel. Das wesentliche Merkmal dieser société civile, welche in der späteren deutschen Übersetzung als nicht näher spezifizierte „Gesellschaft“ firmiert, sind Klassengegensätze. Erst aber die von der Bourgeoisie durchgeführte bürgerliche Revolution, so Marx, habe aus der alten „société“ eine „société bourgeoise“ gemacht. ${ }^{16}$ Diese bilde nun die Basis der bürgerlichen Herrschaft: Die Sphäre der kapitalistischen Produktion, auf dem egoistische Individuen den naturzuständlichen der Krieg aller gegen aller fortsetzen und sich als Kapitalisten ungehindert den durch die Arbeit des Proletariats erzeugten Mehrwert aneignen können.

Emanzipation habe die bürgerliche Revolution nur in Form „politischer Emanzipation“ und „Bürgerrechten“ im Überbau verwirklicht, d.h. im politischen Staat. ${ }^{17}$ Dieser setzt sich zusammen aus den mit Rechten ausgestatteten Bürgern und einer mit Autorität ausgestatteten Regierung. Von dieser politischen Emanzipation bleibe das Proletariat jedoch ausgeschlossen - denn Marx ging hier noch davon aus, dass sich die Bourgeoisie den Forderungen der Arbeiterschaft nach allgemeinem Wahlrecht auf lange Sicht widersetzt. $\mathrm{Zu}$ einer vollständig emanzipierten Gesellschaft, in welcher der Naturzustand auch in der Sphäre der Produktion aufgehoben ist, komme es dem zu Folge erst nach einer proletarischen Revolution, welche an die Stelle der alten „société civile“ eine „association“ setzt, in der alle Klassengegensätze aufgehoben sind. ${ }^{18}$

Als 1888 die deutsche Übersetzung Das Elend der Philosophie erschien, hatten Eduard Bernstein und Karl Kautsky, redaktionell betreut von Friedrich Engels, jedoch société civile als Gesellschaft und société bourgeoise als bürgerliche Gesellschaft übersetzt. ${ }^{19}$ Mit dieser Unterschlagung des Zivilen haben die Übersetzer einen Einschnitt in der deutschsprachigen Theoriegeschichte vorgenommen. Der Begriff der bürgerlichen Gesellschaft, der von Hegel als Entsprechung für das Lockesche civil society ins Deutsche eingeführt wurde, war nun zur Entsprechung der Marxschen société bourgeoise geworden. Die Marxsche società civile, die sowohl Elemente Hegels bürgerlicher Gesellschaft als auch Lockes civil society enthielt und von Gramsci als società civile wieder aufgegriffen wurde, ging im Deutschen verloren. Ihre Wiederentdeckung Ende der 1980er Jahre erforderte daher ein Ausweichen auf einen Alternativbegriff, als der sich inzwischen Zivilgesellschaft etabliert hat.

Der begriffsgeschichtliche Ausgangspunkt der „Zivilgesellschaft“, Gramscis società civile, ist - anders als die société civile des jungen Karl Marx - kein

\footnotetext{
16 Marx 1968 [1847]: 67-68.

17 Marx 1976 [1844].

18 Marx 1968 [1847]: 68.

19 Marx 1968 [1847], 1972 [1888].
} 
Oberbegriff. Gramsci gebrauchte società civile zur Bezeichnung eines Teilsystems innerhalb des ,integralen Staats“, welcher die größte Kategorie seiner politischen Theorie darstellt - vergleichbar der sittlichen Substanz bei Hegel und der société civile bei Marx. Der integrale Staat enthält neben der Zivilgesellschaft die Sphäre der politischen Gesellschaft. Gramsci reaktivierte also Begriffe, die schon bei John Locke auftauchen. Nur dass letzterer sie noch synonym und damit näher am Original verwendete - denn die Attribute politisch und zivil sind äquivalent, erstes ist griechischen und zweites lateinischen Ursprungs. ${ }^{20}$ Bei Gramsci jedoch bilden sie unterschiedliche Aspekte des Gemeinwesens.

Die politische Gesellschaft (società politica) ist bei Gramsci die Sphäre der mit Sanktionsgewalt ausgestatteten politischen Institution. Also die Judikative, Legislative und Exekutive sowie das von diesen drei Gewalten hervorgebrachte und erzwungene positive Recht. Zentrales Moment der politischen Gesellschaft ist der Zwang; ihre zentralen Akteure sind Bürokraten. Die Zivilgesellschaft hingegen versteht Gramsci als Sphäre der Überzeugungen, Meinung, Werte und Weltanschauungen. Diese ist juristisch indifferent, d.h. frei von politischem Zwang. Auch die Zivilgesellschaft verfügt über Institutionen. Im bürgerlichen Staat sind dies vor allem Schulen, Kirchen, Medien, freiwillige Zusammenschlüsse und Parteien, welche Überzeugungen, Meinungen, Weltanschauungen hervorbringen und kultivieren. Zentrales Moment der Zivilgesellschaft ist die Hegemonie; ihre zentralen Akteure nennt Gramsci Intellektuelle.

Die Zivilgesellschaft Gramscis ist zwar frei von Zwang, nicht aber frei von Herrschaft und Klassengegensätzen. Im bürgerlichen Staat sei es der Bourgeoisie gelungen, ihre Werte und Weltanschauung fest in der Zivilgesellschaft zu verankern, die damit ein Bollwerk gegen gewaltsame Umstürze bildet. Die Institutionen der Zivilgesellschaft erziehen die Angehörigen aller Klassen zur freiwilligen Unterwerfung unter die kapitalistische Produktionsweise. Eine Emanzipation vom Kapitalismus und die Aufhebung von Klassengegensätzen kann daher in entwickelten Staaten nur durch den langsamen Prozess der intellektuellen Hegemoniegewinnung erfolgen. Kommunistinnen müssten sich also auf einen langwierigen „Stellungskrieg“ in der Zivilgesellschaft einstellen, bei dem die Emanzipation von der Herrschaft der Bourgeoisie und die Errichtung einer klassen- und zwanglosen Gesellschaft, d.h. eines „ethischen Staates“, in unabsehbare Ferne rücken.

Die Funktionen der beiden Sphären des Staates bringt Gramsci in der Formel „Staat = politische Gesellschaft + Zivilgesellschaft, das heißt Hegemonie, gepanzert mit Zwang“ auf den Punkt. ${ }^{21}$ Institutionen, die im bürgerlichen Staat reproduktive und produktive Funktionen erfüllen, wie Familien und Fabriken,

20 Locke 1999: 139; Pijl 1996: 61. 
sind für Gramsci nicht von theoretischem Interesse, bzw. stellt für ihn die Familie, anders als für Hegel kein gesellschaftskonstituierendes Element dar.

Als Hirata Kiyoaki 1948 und damit lange vor seiner Beschäftigung mit Gramscis Konzepten Marx' Das Elend der Philosophie ins Japanische übersetzte, bemerkte er die Diskrepanz zwischen französischem Original und deutscher Übersetzung. ${ }^{22}$ Wie das Deutsche kennt aber auch das Japanische keine Unterscheidung zwischen civile und bourgeoise. Das Problem löste Hirata, indem er société bourgeoise als burujoa shakai und société civile als shimin shakai übersetzte. ${ }^{23}$ Die Technik, Fremdworte durch Umschrift in Katakana zu japanisieren, erlaubte es ihm, die Unterscheidung aufrechtzuerhalten. Es ist Hirata hoch anzurechnen, dass er sich bei der Übersetzung am französischen Original orientierte, denn so blieb der japanischen Nachkriegsdebatte eine radikale Umdeutung der bürgerlichen Gesellschaft (shimin shakai), bzw. eine Verwechslung von shimin (Bürgerin) und burujoa (Bourgeois) weitgehend erspart.

Deshalb benötigt Hirata auch keinen Alternativbegriff $\mathrm{zu}$ shimin shakai, wenn er im zweiten Teil seines Aufsatzes die società civile Gramscis übersetzt, welche eine Weiterentwicklung Hegels bürgerlicher Gesellschaft und Marx' société civile ist, nicht aber seiner société bourgeoise. So schrieb Haug 1988:

Nur wenn man Marx’ Rede von der »bürgerlichen Gesellschaft" mit civil society übersetzt statt mit bourgeois society [hier kehrt Haug die historische Richtung der Übersetzung um], kann man sagen, daß Marx und Gramsci den selben Term benutzen für unterschiedliche Gehalte, den Unterschied im Sprachgebrauch zur gegensätzlichen Sachauffassung steigernd. Dasselbe Objekt scheint dann für Marx zur Basis, für Gramsci zum Überbau zu gehören. ${ }^{24}$

Den Ursprung jener Verwechslung entdeckte Hirata, das Ausmaß des daraus resultierenden „hermeneutischen Schlamassel[s]” ${ }^{25}$ war ihm wohl nicht bewusst. Da sich die bürgerliche Gesellschaft im Deutschen fest als Entsprechung der société bourgeoise etabliert hatte, musste die deutsche Gramsci-Übersetzung auf einen Alternativbegriff ausweichen, um Gramscis società civile von dieser unterscheidbar zu machen. 1988 schlug Wolfgang Fritz Haug vor, den Begriff „der Sprache etwas Neues beibringend [...] als zivile Gesellschaft“26 $\mathrm{zu}$ übersetzen. 1989 legten sich er und andere auf Zivilgesellschaft fest.

Es würde aber zu noch größerem Schlamassel führen, übersetzten wir nun Hiratas shimin shakai von 1989 rückwirkend als Zivilgesellschaft. Aus diesem

21 Gramsci et al. 1991-2002: H6 § 88, 783.

22 Hirata 1989: 122.

23 Hirata 1989: 122.

24 Haug 1988: 34.

25 Haug 1988: 33.

26 Haug 1988: 43 
Grund haben wir uns entschieden, Hiratas sprachliches Manöver nachzuspielen. Wir übersetzen shimin shakai (société civile/società civile) konsequent als bürgerliche Gesellschaft, egal ob es sich dabei um den Hegelschen, Marxschen oder Gramscischen Begriff handelt. Das Japanische burujoa shakai (société bourgeoise/società borghese) soll dementsprechend als bourgeoise Gesellschaft übersetzt werden. Wir tun damit so, als hätte es die deutsche Übersetzung von Das Elend der Philosophie nie gegeben. Nur ein einziges Mal taucht in dieser übrigens die „Bourgeoisgesellschaft” auf. ${ }^{27}$ Das Wort erscheint uns daher nicht nur ungeeignet als Gegenstück zur bürgerlichen Gesellschaft sondern als ideengeschichtlich irrelevant, weshalb wir es nicht benutzen.

\subsection{Die Bedeutung des Textes für die Ideengeschichte}

Hiratas Entdeckung, dass Marx 1847 zwischen société civile und société bourgeoise unterschieden hatte, hat bis heute keinen Nachhall gefunden. Weder in der japanischen, deutschen oder englischen Begriffsgeschichte wurde seine Beobachtung zur Kenntnis genommen. Genauso verhält es sich mit seinem eigenen Beitrag zur civil society (shimin shakai) Debatte nach $1990 .^{28}$ Dies ist besonders bemerkenswert, da es anders als im deutschsprachigen Raum im Japanischen mit Hirata einen marxistischen Intellektuellen gab, der sich die ganze Nachkriegszeit hindurch intensiv mit der bürgerlichen Gesellschaft als Methodenbegriff auseinandersetzte.

Obwohl es also in Japan bereits vor dem civil society hype der 1990er Jahre eine aktive intellektuelle Auseinandersetzung mit dem Begriff shimin shakai gab und diese, anders als in Deutschland, auch in der Folge mit dem gleichen Begriff weitergeführt wurde, hatte die ältere keinen großen inhaltlichen Einfluss auf die jüngere Debatte. ${ }^{29}$ Es kam damit auch in Japan zu einer „Übertragung auf einen anderen Sachverhalt““30 Anders als Simon Avenell suggeriert, ist der japanischsprachige Beitrag zum Begriff der „civil society“ (shimin shakai) in der Nachkriegszeit (vor 1990) daher auch keine Pionierleistung in Bezug auf die Wiederbelebung des Begriffs in den 1990er Jahren. ${ }^{31}$ Der Beitrag Hiratas und anderer marxistischer Intellektueller in Japan wie in Europa von vor 1990 wurde vom hegemonialen Diskurs ab ca. 1990 völlig ignoriert (oder fehlinterpretiert).

27 Marx 1972 [1888], 2, § 5: 181.

28 Vgl. Avenell 2011.

29 Vgl. Foljanty-Jost/Haufe 2006.

30 Haug 1994: 494.

31 Avenell 2011: 333; vgl. auch Seifert 1999: 23. 
Der rote Faden der Ideengeschichte der bürgerlichen Gesellschaft wurde in dem Moment ein zweites Mal abgetrennt, als Jürgen Habermas den Begriff Zivilgesellschaft hijackte, um ihn für etwas anderes $\mathrm{zu}$ benutzen als er eigentlich vorgesehen war, d.h. nicht als Übersetzung der Gramscischen società civile in Abgrenzung zur società politica, sondern in einem engeren und normativ überfrachteten Sinne, als Abgrenzung zu den stärker „vermachteten“ Bereichen der Öffentlichkeit, d.h. dem Lobbyismus etablierter Verbände und Parteien einerseits sowie den professionalisierten Medien andererseits. ${ }^{32}$ Habermas verlangt von den Akteuren der Zivilgesellschaft übermenschliches, nämlich, dass sie ausschließlich „problemlösend“ agieren und sich in „Selbstbegrenzung“ üben, also nicht bloß ihre Interessen vertreten und auf Macht verzichten. ${ }^{33}$

Dem Habermasschen Beispiel folgend und dieses weiter vulgarisierend, benutzen die meisten Autor^innen den Begriff Zivilgesellschaft (civil society /shimin shakai) ab ca. 1990 neo-tocquevillianisch, d.h. als Abgrenzungsbegriff zu den liberal (also unkritisch und ahistorisch) definierten Bereichen Familie, Markt und Staat sowie normativ als Aufforderung zur Kritik an Regierungen und Geschäftspraktiken von links - dann oft mit Bezug auf Habermas oder einen falsch verstandenen Gramsci - oder aber zum freiwilligen Engagement in Vereinen und der Kirche von rechts - mit Bezug auf Dahrendorf in Europa und Putnam in den USA und Japan sowie einem rein legitimatorischen Rückgriff auf einen falsch verstandenen und fehlerhaft übersetzten Tocqueville. ${ }^{34}$ Daran konnte auch die Wiederentdeckung und Übersetzung der Gefängnishefte Gramscis wenig ändern. Der bis hier nachvollzogene Bedeutungswandel ist in Tabelle 1 veranschaulicht.

Im nun folgenden ersten Textteil zeigt Hirata, dass Marx Hegels bürgerliche Gesellschaft in société civile und société bourgeoise aufspaltet und den (politischen) Staat der société civile d.h. der Gesellschaft unterordnet. Darauf aufbauend legte Hirata 1989 dar, wie Gramsci den Staat Hegels als „intergralen Staat“ befreit von monarchistischen Vorzeichen und weltgeistlicher Metaphysik ${ }^{35}$ wieder als zentralen Begriff des Überbaus einführt, den er aus bürgerlicher Gesellschaft bzw. Zivilgesellschaft und politischer Gesellschaft zusammensetzt. Wie bei Marx bildet auch bei Gramsci die bourgeoise Gesellschaft (società

32 Habermas 1992: 453-458.

33 Habermas 1992: 443, 449; vgl. auch Markner 1994.

34 Vgl. Edward/Foley 1998; Edwards et al. 2001; Haug 1994: 493; Kessl 2011: 1769; Zaleski 2008: 260. 35 In den GPR entwarf Hegel eine teleologische Hierarchie sozialer Ordnungen. Die primitivste Form sozialer Organisation sei die Familie. Aus dieser hervor ginge im Laufe der historischen Entwicklung die bürgerliche Gesellschaft, welche dann zur höchstentwickelten Form des Gemeinwesens, dem Staat, führen kann (Horstmann 2005: 204; Bourgeois 2005: 224). Am besten verwirklicht sah Hegel diesen in der konstitutionellen Monarchie. Hegel personifizierte Staaten, 
Tabelle 1: Bedeutungswandel und Übersetzungen der bürgerlichen Gesellschaft.

\begin{tabular}{|c|c|c|c|c|c|}
\hline \multirow{3}{*}{$\begin{array}{l}\text { Hegel, } \\
\text { Gans }\end{array}$} & \multicolumn{5}{|c|}{ Sittlichkeit / sittlicher Geist / sittliche Substanz } \\
\hline & Familie & \multicolumn{3}{|c|}{ bürgerliche Gesellschaft } & Staat \\
\hline & $\begin{array}{c}\text { natürlicher } \\
\text { Geist } \\
+ \text { Subjekt }\end{array}$ & $\begin{array}{c}\text { A: } \\
\text { System der } \\
\text { Bedürfnisse } \\
\text { Berufsstände, } \\
\text { Markt }\end{array}$ & $\begin{array}{c}\text { B: } \\
\text { Rechtspflege } \\
\text { Schutz des } \\
\text { Eigentums }\end{array}$ & $\begin{array}{c}\text { C: } \\
\text { Polizei und } \\
\text { Korporation } \\
\text { Bürokratie, } \\
\text { Wohlfahrt, } \\
\text { Vereinigungen }\end{array}$ & $\begin{array}{l}\text { höchstes Recht } \\
\text { politisches } \\
\text { Gemeinwesen, } \\
\text { öffentliche } \\
\text { Meinung, } \\
\text { Exekutive, } \\
\text { Legislative }\end{array}$ \\
\hline \multirow{8}{*}{$\begin{array}{l}\text { Marx } \\
\text { (Engels, } \\
\text { Bernstein, } \\
\text { Kautsky) }\end{array}$} & \multicolumn{5}{|c|}{$\begin{array}{l}\text { (kapitalistischer) Staat / Gesellschaft (société civile) } \\
\text { repräsentative Demokratie = politische Emanzipation }\end{array}$} \\
\hline & \multicolumn{3}{|c|}{ Basis } & \multicolumn{2}{|c|}{$\ddot{U}$ berbau } \\
\hline & \multicolumn{3}{|c|}{ Produktionsverhältnisse } & \multicolumn{2}{|c|}{ Herrschaft / Rechtsordnung } \\
\hline & \multicolumn{3}{|c|}{$\begin{array}{l}\text { bürgerliche Gesellschaft } \\
\text { (société bourgoise) }\end{array}$} & \multicolumn{2}{|c|}{ politischer Staat } \\
\hline & \multicolumn{3}{|c|}{ Menschenrechte } & \multicolumn{2}{|c|}{ Bürgerrechte } \\
\hline & \multicolumn{3}{|c|}{ Privatrecht (Krieg aller gegen alle) } & \multicolumn{2}{|c|}{ öffentliches Recht } \\
\hline & \multicolumn{3}{|c|}{ Eigentum, Besitz, Bildung, Religion } & meinwesen & Autorität \\
\hline & \multicolumn{3}{|c|}{ (egoistischer) Mensch, Bourgeois } & Bürger & Regierung \\
\hline \multirow[t]{6}{*}{ Gramsci } & \multirow[t]{5}{*}{$\ddot{U}$ Uerbau } & \multicolumn{4}{|c|}{ juristisch indifferent $\leftarrow$ Integraler Staat $\rightarrow$ juristisch verbindlich } \\
\hline & & \multicolumn{2}{|c|}{$\begin{array}{l}\text { Zivilgesellschaft } \\
\text { (società civile) }\end{array}$} & \multicolumn{2}{|c|}{$\begin{array}{l}\text { politische Gesellschaft } \\
(\text { società politica })\end{array}$} \\
\hline & & \multicolumn{2}{|c|}{ lltagsverstand, Meinung, Normen } & \multicolumn{2}{|c|}{ Gesetze, positives Recht } \\
\hline & & \multicolumn{2}{|c|}{$\begin{array}{l}\text { Kirche, Schule, Medien, } \\
\text { Wissenschaft, Vereinigungen }\end{array}$} & \multicolumn{2}{|c|}{ Judikative, Legislative, Exekutive } \\
\hline & & \multicolumn{2}{|c|}{ Intellektuelle $\rightarrow$ Hegemonie } & \multicolumn{2}{|c|}{ Beamte $\rightarrow$ Zwang } \\
\hline & Basis & \multicolumn{4}{|c|}{ Produktions- und Reproduktionsverhältnisse } \\
\hline \multirow[t]{6}{*}{ Habermas } & \multicolumn{5}{|c|}{ Gesellschaft / Rechtsstaat } \\
\hline & \multicolumn{4}{|c|}{ Gesellschaft } & Staat \\
\hline & \multicolumn{2}{|l|}{ Lebenswelt } & \multicolumn{3}{|c|}{ Politische Öffentlichkeit } \\
\hline & Privatsphär & nicht-verma & $\begin{array}{l}\text { Öffentlichke } \\
\text { itet (autochth }\end{array}$ & /vermachtet & $\begin{array}{c}\text { politisches } \\
\text { Zentrum }\end{array}$ \\
\hline & Individuen & Zivilgesellschaft & Medien & Lobbyismus & Gewaltmonopol \\
\hline & & & Sprache / Di & & \\
\hline
\end{tabular}

Eigene Darstellung nach Bourgeois 2005; Gramsci/Bochmann/Haug 1991-2002; Habermas 1992; Hegel 1981; Horstmann 2005; Marx 1968 [1847], 1972 [1888], 1976 [1844].

borghese) die ökonomische Basis. Wie dies im Einzelnen von Hirata ausgelegt wurde, wird in der noch anstehenden Übersetzung des zweiten Teils des hier besprochenen Aufsatzes zu zeigen sein.

in dem er diesen einen inhärenten „Volksgeist“ zuspricht. In der Weltgeschichte vereinigten sich diese dann zum „Weltgeist“ (Pijl 1996: 76). 


\section{2 Übersetzung}

„Über Gramscis Begriff der bürgerlichen Gesellschaft (Teil 1)“

I. Hegel und Marx im Begriff der bürgerlichen Gesellschaft

I. a) Einleitung - Die Bedeutung des Lesens

Liest man eine Schrift nicht vorurteilsfrei, wird man sie nicht wirklich gelesen haben. Doch würde die Handlung des Lesens nicht auskommen ohne ein Verlangen nach einem ideellen Rahmen, welcher Ort und Weise des eigenen Lebens in gewissem Sinne ausleuchtet. Es gibt nur zwei wesentliche Arten des Lesens: heuristisches (heuristique) Lesen und problematisches (problematique) Lesen als Infragestellung des Selbst. Das Lesen ist damit eine subjektive Einmischung in die Wirklichkeit. Als sich Louis Althusser (1918-1990) seinem Werk „Das Kapital“ lesen widmete, war dies eine philosophische Einmischung in die ganz Frankreich umfassende kapitalistische Kultur, durch die Darlegung der Lesart einer einzelnen Schrift. ${ }^{36}$

Als ich 1968 „Bürgerliche Gesellschaft und Sozialismus“37 veröffentlichte, hat dies eine Resonanz erzeugt, mit der ich nicht gerechnet habe. Es wurde vielfach gelesen und kommentiert. Ich als Autor befürchtete damals weder die notwendige Reichweite, noch mein Ziel erreichen zu können.

Dies hatte einen historischen Hintergrund. Die Spuren der Nachkriegsrevolution wurden von einem Vorgefühl der hereinbrechenden Krise der 1970er Jahre überlagert. Marx ist auf eine der Zeit eigene Weise gelesen und interpretiert worden. Dabei wurde auch meine Lesart, der man das Etikett ,Theorie der bürgerlichen Gesellschaft‘ angehängt hatte, zum Gegenstand der Diskussion und rief ab und zu Kritik hervor.

Heute, in der zweiten Hälfte der 1980er Jahre, befinde ich mich in einem Japan, welches sich von Marx abgewandt hat. In einem Japan des ausgehenden Jahrhunderts, dessen ,Nachkriegszeit vorbei ist ${ }^{48}$ und dessen Hochmut als ,Wirtschaftsmacht` in der Welt Stirnrunzeln hervorruft, während es im Innern

36 Lire le Capital, erschienen 1965, vgl. Althusser 1996. Die Althusserische Interpretationsweise wird u.a. dafür kritisiert, dass sie den ersten Abschnitt des Kapitals, in dem Marx das Verhältnis von „Ware und Geld“ beschreibt, völlig außer Acht gelassen bzw. „zensiert“ habe, vgl. Liepitz 1992: 22; sowie Grefrath 2016.

37 Shimin shakai to shakaishugi, vgl. Hirata 1969; sowie Seifert 1999: 23.

38 Mohaya sengō de ha nai (Formulierung aus dem 1956er Wirtschaftsweißbuch des Wirtschaftsplanungsbüros [Keizai Kikakuchō], heute Kabinettsbüro [Naikakufu]). 
durch eine ,Epoche der Ungewissheit ${ }^{39}$ in einen Ozean des Agnostizismus stürzt.

Während meiner Zeit als Gastprofessor an der (siebten und dritten) Universität von Paris diskutierte ich häufig mit verschiedenen französischen Freunden über das heutige Japan. Dabei wurde mir bewusst, dass unter den verschiedenen Aspekten des Gegenwartskapitalismus, für den die Dominanz multinationaler Konzerne charakteristisch ist, ein Thema existiert, welches „die räumlichen Sphären der bürgerlichen Gesellschaft der Gegenwart“40 genannt werden sollte.

In der Gestalt von multinationalen Unternehmen entwickeln sich die Zirkulationsprozesse des Produktionskapitals vor einem globalen Horizont und einem nationalen Wasserstand. Dies befördert einerseits die Gestaltung neuer und originärer sozialer Räume und lässt andererseits neue politik- und sozialwissenschaftliche Problemfelder entstehen. Gleichzeitig spürte ich, dass dies einen tiefgreifenden Impact auf die politische Ökonomie hatte. Mir dieses Gedankens bewusst werdend, habe ich ein Diskussionspapier mit dem Titel „Die politische Ökonomie des Gegenwartskapitalismus (Prolog) ${ }^{“ 41}$ in Shisō (vom August 1987) veröffentlicht.

Je mehr ich dem gegenwärtigen Japan anhafte, gleichwohl ich dieses mehr oder weniger von außen beobachte, umso mehr empfinde ich die Notwendigkeit eines „Bezugspunktes“ (shiza 視座), der sich als Gemeinsamkeit durch unterschiedliche Epochen hindurch zieht. - Die gesellschaftlichen Verhältnisse einer bestimmten Epoche lassen sich nicht allein auf die wirtschaftlichen Produktionsverhältnisse $^{42}$ zurückführen. Während sie durch die letzteren beschränkt und konditioniert werden, haben sie doch die Möglichkeit zur selbständigen Entwicklung. Ihre eigentümliche Modalität wiederum liefert den epochenspezifischen Rahmen, welcher zwischen den Produktionsverhältnissen und den Produktionskräften vermittelt. Somit sind gerade die Widersprüche der rhythmisierten Produktionskräfte und -verhältnisse die gemeinsame Grundlage von Umwälzungsprozessen der staatlichen Dimension ....

Lässt sich dieser Gedanke nicht schon bei Marx im Begriff „bürgerliche Gesellschaft“43 in Bruchstücken erahnen? Mit dieser Überlegung habe ich den

39 Fukakujissei no jidai (von John Kenneth Galbraith kreierte Fernsehserie der BBC aus dem Jahre 1977 „The Age of Uncertainty“).

40 Gendai shimin shakai no kūkanteki shoken'iki 現代市民社会の空間的諸領域.

41 „Gendai shihonshugi no seiji keizaigaku (joshō)“, vgl. Hirata 1987b.

42 Seisan shokankei 生産諸関係.

43 Shimin shakai 市民社会. 
Artikel „Der Kapitalismus der Gegenwart und die bürgerliche Gesellschaft“ (in Hirata/Yamada/Yagi [Hrsg.] [1987]: Kreislauf der bürgerlichen Gesellschaft der Gegenwart, Shōwadō) ${ }^{44}$ verfasst.

Mit der Veröffentlichung der beiden oben genannten Artikel habe ich mich, während ich gleichzeitig auf Marx’ Das Kapital zurückschaute, an einer wissenschaftsgeschichtlichen Erläuterung versucht, die sich mit der Verbindung seiner ,Ökonomie ${ }^{645}$ und seiner ,Politikwissenschaft ${ }^{\star 46}$ befasst.

Mir bestätigte sich erneut, dass der Gebrauch von Marx erst vermittelt durch eine Re-Immanenz ${ }^{47}$ von Marx verwirklicht wird.

In diesem Prozess begegnete ich erneut den klassischen Themen bürgerliche Gesellschaft und Staat.

Zum einen liegt hier ein ideelles Theorieproblem vor, das mit der Beziehung zwischen Hegel und Marx zu tun hat. Ersterer sah die Aufhebung der bürgerlichen Gesellschaft im Staat vor. Letzterer stellte eine Aufhebung von Staat und bürgerlicher Gesellschaft in Form eines Wiederaufgehens des Staates in der bürgerlichen Gesellschaft in Aussicht. Gleichzeitig besteht hier aber auch ein ideen- und bewegungsgeschichtliches Problem, welches sich in der Auseinandersetzung zwischen Proudhon und Marx um die Unterscheidung und den Zusammenhang von sozialen und politischen Bewegungen verbirgt. Ich glaube daher, dass jene klassischen Begriffe bürgerliche Gesellschaft und Staat auch mit den bewegungstheoretischen Problemstellungen des gegenwärtigen Japans in Verbindung stehen.

Soweit es der Umfang dieses Aufsatzes erlaubt, werde ich hier zunächst, wozu ich bisher nicht die Gelegenheit hatte, meine Begegnung mit dem Begriff der bürgerlichen Gesellschaft bei Marx schildern. Daran anschließend werde ich die zum Ende des Jahrhunderts und 50 Jahre seinem Tod neu erlangte, sozialund geschichtswissenschaftliche Bedeutung Antonio Gramscis aufzeigen.

\section{I.1 Der Begriff „bürgerliche Gesellschaft“ in Das Elend der Philosophie - Unterscheidung und Zusammenhang von société bourgeoise und société civile}

In den Jahren 1948 und 1949 hatte ich Das Elend der Philosophie [aus dem Französischen] ins Japanische übersetzt. Dabei ,entdeckte‘ ich, dass in dessen

44 „Gendai shihonshugi to shimin shakai“, vgl. Hirata 1987c.

45 Keizai gaku puran 経済学プラン.

46 Seiji gaku puran 政治学プラン.

47 Sainaizai 再内在. 
letzten Abschnitten société bourgeoise und la société civile unterschiedlich gebraucht werden.

Marx, der kritische Nachfolger Hegels, der „bourgeois“48 (Privatmensch [shijin 私人]) und „citoyen”49 (Staatsbürger [kōmin 公民]) unterschiedlich auffasste, hat jenen zwei Begriffen verschiedene Bedeutungen gegeben und darüber hinaus die beiden französischen Vokabeln im Sinne eines gesellschaftskritischen Selbstverständnisses klar getrennt. Es ist daher auch nichts Mysteriöses daran, den Zusammenhang der beiden zu diskutieren.

Im von ihm verwendeten Deutschen jedoch fehlte das Vokabular, um die beiden zu unterscheiden. Im Deutschen hat Marx, wie auch schon Hegel, beide gleichermaßen als die bürgerliche Gesellschaft [deutsch im Original] ausgedrückt. ${ }^{50}$

Im Französischen hingegen sind die beiden, auch wenn zwischen ihnen ein Zusammenhang besteht, deutlich unterschieden. Marx war sich dessen bewusst und verwendete daher diese zwei Termini in Das Elend der Philosophie entsprechend.

Daher müssen bei einer Übersetzung auch unterschiedliche Übersetzungsbegriffe verwendet werden. Ich habe also, mehr oder weniger von Zögern begleitet, ersteren als „bourgeoise Gesellschaft“51 und letzteren als „bürgerliche Gesellschaft“52 übersetzt. Letzterer enthält, ähnlich dem Begriff der „Staatsbürgergesellschaft“, 53 einen bedeutenden Aspekt im Punkt der Verhältnisse zum Staat. Aber in Anbetracht dessen, dass Marx’ Begriff des „Staatsbürgers/ citoyen “54 mehr als bei Hegel eine reale soziale Bestimmtheit mit einschließt, habe ich mich letztendlich für „bürgerliche Gesellschaft“ entschieden. -Dieser Punkt verdient besondere Beachtung, da er mit jener ökonomischen Trinitätsformel zusammenhängt, die beim späten Marx von gesamtheitlicher Bedeutung ist.

Nun aber da ich Übersetzungen veröffentlicht sehe, welche sich für den Übersetzungsbegriff „shimin shakai“ entschieden haben (1950 Marx Engels Sammlung ausgewählter Schriften [Marukusu-Engurusu senshū], Band 1), schmerzt mir die Brust. Der Übersetzungsbegriff „shimin shakai“ lässt einen an

48 Burujoa ブルジョア.

49 Shitowaiyan シトワイヤン.

50 Hier irrt Hirata. Er lässt nicht nur unberücksichtigt, dass die Übersetzung nicht von Marx selbst, sondern von Eduard Bernstein und Karl Kautsky stammt, auch übergeht er, dass diese i. d.R. nur société bourgeoise als bürgerliche Gesellschaft übersetzten, während sie aus der société civile meist eine nicht näher attributierte Gesellschaft machten.

51 Burujoa shakai ブルジョア社会.

52 Shimin shakai 市民社会.

53 Kōmin shakai 公民社会.

54 „Staatsbürger“ (kōmin 公民) in Kanji mit „citoyen“ (shitowaiyan シトワイヤン) als Furigana. 
die bürgerliche Gesellschaft [deutsch im Original] im Deutschen denken. Zu leicht kann hier die Unterscheidung von „bourgeoise Gesellschaft“ und „bürgerliche Gesellschaft“ verschwinden. Um sicher zu gehen, möchte ich jene Übersetzung an dieser Stelle zitieren.

„Mit Bezug auf die Bourgeoisie (den Bürgerstand) haben wir zwei Phasen zu unterscheiden: die, während derer sie sich unter der Herrschaft des Feudalismus und der absoluten Monarchie als Klasse konstituierte, und die, wo sie, bereits als Klasse konstituiert, die Feudalherrschaft und die Monarchie umstürzte, um die Gesellschaft zu einer Bourgeoisgesellschaft ${ }^{55} \mathrm{zu}$ gestalten ... “56

„Die arbeitende Klasse wird im Laufe der Entwicklung an die Stelle der alten bürgerlichen Gesellschaft ${ }^{57}$ l'ancienne société civile eine Assoziation une association setzen, welche die Klassen und ihren Gegensatz ausschließt, und es wird keine eigentliche politische Gewalt mehr geben, weil gerade die politische Gewalt der offizielle Ausdruck des Klassengegensatzes innerhalb der bürgerlichen Gesellschaft ist.“

„Man sage nicht, daß die gesellschaftliche Bewegung die politische ausschließt. Es gibt keine politische Bewegung, die nicht gleichzeitig auch eine gesellschaftliche wäre“

(Karl Marx: Misère de la Philosophie, la Réponse à la Philosophie de la Misère de M. Proudhon, Tokyo Aoki Shoten, 1982: 176-177; japanische Übersetzungen Marukusu keizaigaku tetsugaku ronshū, Gendai no daishisō II.4., Kawade Shobō: 436-437. Marukusu Engerusu zenshū 4, Ōtsuki Shoten: 189-190). ${ }^{58}$

Dans la bourgeoisie, nous avons deux phases à distinguer: celle pendant laquelle elle se constitua en classe sous le régime de la féodalité et de la monarchie absolue, et celle où, déjà constituée en classe, elle renversa la féodalité et la monarchie, pour faire de la société une société bourgeoise. La première de ces phases fut la plus longue et nécessita les grands efforts. Elle aussi avait commencé par des coalitions partielles contre les seigneurs féodaux.

On a fait bien des recherches pour retracer les différentes phases historiques que la bourgeoisie a parcourues, depuis la commune jusqu'à sa constitution comme classe.

Mais quand il s'agit de se rendre un compte exact des grèves, des coalitions et des autres formes dans lesquelles les prolétaires effectuent devant nos yeux leur organisation comme classe, les uns sont saisis d'une crainte réelle, les autres affichent un dédain transcendental.

55 Bei Hirata „einer bourgeoisen Gesellschaft“ (hitotsu no burujoa shakai une société bourgeoise).

56 Aus der deutschen Übersetzung von Das Elend der Philosophie. Hervorhebungen und Ergänzungen bei Hirata 1989: 122.

57 Eine der seltenen Stellen in Das Elend der Philosophie bei denen société civile als bürgerliche Gesellschaft übersetzt wurde.

58 Aus der deutschen Übersetzung von Das Elend der Philosophie, Hervorhebungen und Ergänzungen nach Hirata 1989: 122-123. An dieser Stelle folgt ein längerer Auszug aus der französischen Originalfassung von 1847. Dieser wird hier vollständig wiedergegeben. Anschließend folgt zum Vergleich die entsprechende Textstelle in der deutschen Übersetzung von Eduard Bernstein und Karl Kautsky aus dem Jahr 1888. 
Une classe opprimée est la condition vitale de toute société fondée sur l'antagonisme des classes. L'affranchissement de la classe opprimée implique donc nécessairement la création d'une société nouvelle. Pour que la classe opprimée puisse s'affranchir, il faut que les pouvoirs productifs déjà acquis et les rapports sociaux existants ne puissent plus exister les uns à côté des autres. De tous les instruments de production, le plus grand pouvoir productif c'est la classe révolutionnaire elle-meme. L'organisation des éléments révolutionnaires comme classe suppose l'existence de toutes les forces productives qui pouvaient s'engendrer dans le sein de la société ancienne.

Est-ce à dire qu'après la chute de l'ancienne société il y aura une nouvell domination de classe, se résumant dans un nouveau pouvoir politique? Non.

La condition d'affranchissement de la classe laborieuse c'est l'abolition de toute classe, de même que la condition d'affranchissement du tiers état, de l'ordre bourgeois fut l'abolition de tous les états et de tous les ordres.

La classe laborieuse substituera, dans le cours de son développement, à l'ancienne société civile une association qui exclura les classes et leur antagonisme, et il n'y aura plus de pouvoir proprement dit, puisque le pouvoir politique est précisément le résumé officiel de l'antagonisme dans la société civile.

(Mit Bezug auf die Bourgeoisie haben wir zwei Phasen zu unterscheiden: die, während derer sie sich unter der Herrschaft des Feudalismus und der absoluten Monarchie als Klasse konstituierte, und die, wo sie, bereits als Klasse konstituiert, die Feudalherrschaft und die Monarchie umstürzte, um die Gesellschaft zu einer Bourgeoisgesellschaft [der Begriff taucht in der deutschen Übersetzung nur ein einziges Mal auf] zu gestalten. Die erste dieser Phasen war die längere und erforderte die größeren Anstrengungen. Auch das Bürgertum hatte mit partiellen Koalitionen gegen die Feudalherrn begonnen.

Man hat viel [sic!] Untersuchungen angestellt, um den verschiedenen historischen Phasen nachzuspüren, welche die Bourgeoisie von der Stadtgemeinde an bis zu ihrer Konstituierung als Klasse durchlaufen hat.

Aber wenn es sich darum handelt, sich genau Rechenschaft abzulegen über die Strikes, Koalitionen und die anderen Formen, unter welchen die Proletarier vor unseren Augen ihre Organisation als Klasse vollziehen, so werden die einen von einer wirklichen Furcht befallen, während die anderen eine transzendentale Geringschätzung an den Tag legen.

Eine unterdrückte Klasse ist die Lebensbedingung jeder auf den Klassengegensatz begründeten Gesellschaft. Die Befreiung der unterdrückten Klasse schließt also notwendigerweise die Schaffung einer neuen Gesellschaft ein. Soll die unterdrückte Klasse sich befreien können, so muss eine Stufe erreicht sein, auf der die bereits erworbenen Produktivkräfte und die geltenden gesellschaftlichen Einrichtungen nicht mehr nebeneinander bestehen können. Von allen Produktionsinstrumenten ist die größte Produktivkraft die revolutionäre Klasse selbst. Die Organisation der revolutionären Elemente als Klasse setzt die fertige Existenz aller Produktivkräfte voraus, die sich überhaupt im Schoß der alten Gesellschaft entfalten konnten.

Heißt dies, dass es nach dem Sturz der alten Gesellschaft eine neue Klassenherrschaft geben wird, die in einer neuen politischen Gewalt gipfelt? Nein. 
Die Bedingung der Befreiung der arbeitenden Klasse ist die Abschaffung jeder Klasse, wie die Bedingung der Befreiung des dritten Standes, der bürgerlichen Ordnung, die Abschaffung aller Stände (4) war.

Die arbeitende Klasse wird im Laufe der Entwicklung an die Stelle der alten bürgerlichen Gesellschaft eine Assoziation setzen, welche die Klassen und ihren Gegensatz ausschließt, und es wird keine eigentliche politische Gewalt mehr geben, weil gerade die politische Gewalt der offizielle Ausdruck des Klassengegensatzes innerhalb der bürgerlichen Gesellschaft ist.

(4) Stände hier im historischen Sinn der Stände des Feudalstaats, Stände mit bestimmten und begrenzten Vorrechten. Die Revolution der Bourgeoisie schaffte die Stände samt ihren Vorrechten ab. Die bürgerliche Gesellschaft kennt nur noch Klassen. Es war daher durchaus im Widerspruch mit der Geschichte, wenn das Proletariat als „vierter Stand“ bezeichnet worden ist.)

Beachtet man beim Lesen dieses Abschnittes die bestimmten und unbestimmten Artikel, sollte folgender Sachverhalt klar werden.

(1) Mit „Bourgeoisie“ ist hier nicht direkt die Klasse der Kapitalisten gemeint, sondern in erster Linie private Individuen, die stark bestimmt sind von Waren und Geldmitteln. Diese [privaten Individuen] betreten die Bühne der Geschichte als persönliche Vermittler der Transformation des Geldes zum Kapital. Während sie sich selbst als Klasse formieren, scheiden sie das Proletariat aus und verwandeln sich selbst in kapitalistische private Individuen. Da ,in dem Maße, wie die Bourgeoisie sich entwickelt, sich in ihrem Schoße auch ein neues Proletariat, ein modernes Proletariat entwickelt“ (op.cit S. 115), gibt es entsprechend im „Lauf seiner Entwicklung [...] zwei Phasen.“

(2) Jene Gesellschaft, welche von der Bourgeoisie beherrscht wird, nachdem diese sich zunächst unter dem „Feudalismus und der absoluten Monarchie“ als eine Klasse herauszubilden begann und anschließend deren Bindungen zerstörend sich zur Klasse formierte, sollte „bourgeoise Gesellschaft” genannt werden. Deren „innere Organisation“ reift zu einer „historischen Produktionsorganisation“, welche auf originelle Weise von der politischen Ökonomie interpretiert werden sollte. Diese immanente kritische Erkenntnis macht die Zukunft („Assoziation-Gesellschaft“ [rengō shakai 連合社会]) als eine durch die inhärenten Widersprüche der Gesellschaft als Ganzem herbeigeführte „eigene Entwicklungsstufe” sichtbar. Daher ist das, was hier bourgeoise Gesellschaft genannt wird, später oft als „Kapitalistengesellschaft” bezeichnet worden.

(3) Demgegenüber ist „die bürgerliche Gesellschaft“ ein Ort, an dem durch „politische Macht“ ein „öffentlicher Zusammenschluss“, d.h. eine politische Zusammenfassung hergestellt wird. Sie ist eine Staatsbürgergesellschaft 
(bzw. eine politische/civil Gesellschaft ${ }^{59}$ - Rousseau), die von inneren „Klassengegensätzen” charakterisiert ist. Es sollte besondere Beachtung finden, dass diese Sphäre sozial und politisch spezifisch ist.

Im Gegensatz zu der oben erwähnten „bourgeoisen Gesellschaft“, welche „eine“ historisch errichtete Gesellschaftsform ist, ist „die bürgerliche Gesellschaft“ ein moderner Gesellschaftsraum, der auf dem Grund der Spannung mit dem politischen Staat steht. Marx hatte ihre Aufhebung als ein solcher Gesellschaftsraum in der Aufhebung des Staates als ihren eigenen Entwicklungsprozess vorhergesehen.

Indem Marx jenen Begriff der bürgerlichen Gesellschaft vorgelegte, hat er jenseits einer Ökonomie, im Sinne einer Anatomie der bourgeoisen Gesellschaft, den Horizont der Staatstheorie im Sinne einer Lehre des kritischen Verstehens der bürgerlichen Gesellschaft aufgestoßen. Damit hat er sich an einer Kritik an Proudhon versucht, der die bloße Notwendigkeit von sozialen Bewegungen erklärt hatte und die Bedeutung von politischen Bewegungen verneinte. Seine Kritik war aber nicht eine bloße Erklärung der Notwendigkeit politischer Bewegungen, sondern vielmehr die Erklärung der grundlegenden Bestimmtheit von politischen Bewegungen durch soziale Bewegungen. Der Vollständigkeit halber hörte Marx in seiner Kritik der politischen Ökonomie nicht bei einer rein begrifflichen Entgegensetzung zu Proudhon auf. Je mehr seine Bemühungen eine Systematik zu errichten voranschritten, wurde seine theoretische Arbeit zu einem Theoriegefüge, welches die Gesamtgestalt der Verdinglichung als Entfremdung in den Blick nahm. Gleichzeitig erhielt sie einen systematischen Charakter als kritische Nachfolge der Hegelschen Rechtsphilosophie. Dieser Streitpunkt ist in den Debatten über den „kapitalistischen Staat“, welche seit der Staatsableitungsdebatte ${ }^{60}$ geführt wurden, umfassend nachgewiesen worden.

59 „Politisch“ (seiji 政治) in Kanji mit „civil“ (shivuru シヴル) als Furigana. Während Gramsci eine deutliche Unterscheidung zwischen politischer und bürgerlicher bzw. Zivilgesellschaft macht, bezieht sich Hirata in dieser Gleichsetzung auf Rousseau bzw. Locke.

60 Zum Verhältnis bürgerlicher Gesellschaft und Staatableitungsdebatte siehe u.a. Deppe 2007: „[...] in der sog. ,Staatsableitungsdebatte“ der 70er Jahre stand das Bemühen um die ,richtige Ableitung` des Staates aus dem ,Kapital` im Zentrum. Diese Debatte verlief sich bald in hoch abstrakte Elaborate, deren Bezug zur wirklichen Bewegung und zu den Kämpfen der Zeit kaum noch nachzuvollziehen war“. Ferner Hirsch 2002: „Die Staatsableitungsdebatte hatte einen explizit politischen Bezugspunkt. Sie richtete sich [...] gegen Illusionen über die Spielräume und Möglichkeiten staatlicher Reformpolitik. [...] Die Staatsableitung ist [...] keine fertige Staatstheorie, sondern [...] die Bestimmung der politischen Form der bürgerlichen Gesellschaft, die in einen umfassenderen theoretischen und historischen Kontext gestellt werden muss. Wenn dies nicht gemacht wird, läuft man [...] in eine theoretische Sackgasse. [...]. Die Ansätze von Gramsci und Poulantzas können mit den Ergebnissen der Staatsableitung in 


\section{I.2 Staat und bürgerliche Gesellschaft bei Hegel und Marx - Versuch einer analytischen Skizze}

Man kann wohl sagen, dass es von Marx aber auch von den ihm folgenden Generationen als Hegels größte Leistung angesehen wurde, dass dieser über die Erkenntnis von Gesellschaft hinaus die Hobbessche und Lockesche „Vermengung“ von bürgerlicher Gesellschaft und Staat einer methodischen Kritik unterzogen hat. Fragt man nach der die beiden verbindenden Überschneidungsfläche, so liegt diese wohl darin, dass [beide] historische Veränderungen ${ }^{61}$ als sozialen Wandel ${ }^{62}$ begriffen haben.

(1) Sich deutlich auf Adam Smith beziehend, gleichwohl den Namen Rousseaus anführend, wies Hegel auf deren gemeinsamen Fehler hin. Jene die bürgerliche Gesellschaft bildenden privaten Individuen lassen der Entwicklung der bürgerlichen Gesellschaft ihren Lauf, während sie durch ihre allseitige Exklusivität im Ergebnis ein „System allseitiger Abhängigkeiten“ bilden. Während die mit der bürgerlichen Gesellschaft verflochtenen gesellschaftlichen Zusammenhänge den privaten Individuen oberflächlich einen gewaltigen Nutzen bringen, werden ihre inneren Zusammenhänge $\mathrm{zu}$ einem Entfremdungszustand zerrissener Einzelner. Daher ist sie selbst ein „oberflächlicher Staat“ und ein „Zwangsstaat“.63

Die bürgerliche Gesellschaft ist aber auch eine Assoziation, ${ }^{64}$ die von Verstandesregeln regiert wird, welche von oberflächlichen Notwendigkeiten durchdrungen sind. Sie ist also auch ein „Verstandesstaat”. ${ }^{65}$ In ihr existiert ein Fundament, auf dem Kants Rationalismus ${ }^{66}$ ruht (bis hier Rechtsphilosophie § 182).

Als der gleichen Ideensphäre angehörend nennt und kritisiert Hegel insbesondere Rousseau, der die „Aufgabe des Staates“ auf „die Sicherung des Eigentums und den Schutz der persönlichen Freiheiten der Person“

\footnotetext{
einiger Hinsicht präzisiert und weiterentwickelt werden. Kurz gesagt, geht es bei Gramsci vor allem um die Analyse hegemonialer Prozesse und das komplexe Verhältnis von Staat und ,ziviler` Gesellschaft. [...]. Als Analyse der politischen Form der bürgerlichen Gesellschaft bleibt die Staatsableitungsdebatte gültig und unverzichtbar. Notwendig ist aber darüber hinaus ein theoretisches Konzept, das es ermöglicht, historische Strukturveränderungen des Kapitalismus und des Staates, konkrete Klassenstrukturen, hegemoniale Prozesse und Krisen zu verstehen“. 61 Rekishi no henka 歴史の変化.

62 Shakai no hendō 社会の変動.

63 Bei Hegel ist von „Not- und Verstandesstaat“ die Rede, vgl. Hegel 1981, GPR § 183: 221.

64 Kyōdōtai 共同体.

65 Gosei kokka 悟性国家.

66 Gosei shugi 悟性主義.
} 
beschränkt und bei der Errichtung eines Staates von einem gesellschaftlichen Einverständnis („Gesellschaftsvertrag“) ausgeht.

Er [Hegel] schätzte Hobbes, Locke, Smith und Rousseau als die Epoche definierende Denker der „Moderne“, verurteilte sie aber auch als Mittäter eines „der Moderne eigenen Trugschlusses“.... es ist wohl erlaubt, dies so zu lesen, als würde Hegel hier plötzlich zu ,Marx‘.

(2) Genauso aber ist Hegels die bürgerliche Gesellschaft [deutsch im Original] identisch mit den von Marx in Misère de la Philosophie gleichzeitig gebrauchten Begriffen „bourgeoise Gesellschaft“ und „bürgerliche Gesellschaft“. Innerhalb der Systementwicklung hat man sich weitgehend an dieser Deutung der orientiert.

Als bourgeoise Gesellschaft ist Hegels bürgerliche Gesellschaft die kapitalistische Gesellschaft auf der von Smith (und Stuart) beschriebenen Stufe. Eine Gesellschaft, in der Arbeitende gleichzeitig selbständige Produzenten und Lohnarbeitende sind, und Bourgeoise zur gleichen Zeit „private Personen“ im Allgemeinen und Kapitalisten im Besonderen sind. In dieser besteht eine Tendenz zur Polarisierung zwischen „exzessivem Reichtum“ und „exzessiver Armut“. Darüber hinaus werden „Person“, „Eigentum“ und „Recht“ zum universellen und ersten Prinzip. Dann wird die „Moral“ als die individuelle Manifestation des freien Willens zum zweiten Prinzip. Diese Prinzipien bereiten die Gründung der bürgerlichen Gesellschaft vor. Je mehr diese Prinzipien innerhalb der bürgerlichen Gesellschaft an Handlungsraum gewinnen und sich entwickeln, desto mehr erhöht sie sich [die bürgerliche Gesellschaft] $\mathrm{zu}$ einer Entwicklungsform des Staates. So schrieb Hegel:

Wie die bürgerliche Gesellschaft der Kampfplatz des individuellen Privatinteresses aller gegen alle ist, so hat hier der Konflikt desselben gegen die gemeinschaftlichen besonderen Angelegenheiten, und dieser zusammen mit jenem gegen die höheren Gesichtspunkte und Anordnungen des Staats, seinen Sitz (Rechtsphilosophie § 289). ${ }^{67}$

Als Ort jenes Gegensatzes und Zusammenstoßes ist die bürgerliche Gesellschaft sowohl die „Sphäre der Bildung“ (birutongu), welche eine „Verfeinerung“ als Sittlichkeit erhält, als auch der Horizont, der sich zum „politischen Staat“

67 Hirata zitiert Hegel in der japanischen Übersetzung wie folgt: „「市民社会は、万人に対し て万人の利益が対立する場であり、個人的利益が共同の特殊的要件と衝突する場であり、こ れら二つのものが一緒になって、国家のより高次な見地と指示とに衝突する場である」

(『法哲学』二八九節) “, vgl. Hirata 1989: 126. Das Zitat stammt aus dem dritten Abschnitt „Der Staat“ des dritten Teils „Die Sittlichkeit“ der GPR, vgl. Hegel 1981: GPR § 289, Notiz: 336. 
entwickeln soll. Folglich, da ihr die oben beschriebene Stellung zukommt, „[gehört] die Schöpfung der bürgerlichen Gesellschaft [...] der modernen Welt an“. ${ }^{68}$

(3) Während die bürgerliche Gesellschaft unaufhörlich ihre Beziehung zum Staat reproduziert, wird sie folgendermaßen skizziert.

a. In der modernen bourgeoisen Gesellschaft steht das „Privateigentum“ [GPR § 46] des Bodens, dem „Vermögen der Gesellschaft“ des städtischen „Handwerksstandes“ [GPR § 204] entgegen. Beide treten gemeinsam in ein Spannungsverhältnis zum „Staatsvermögen“ [GPR $\S 306:$ 352]. Der Gegensatz von arm und reich in der Stadt ist ein Aspekt des Gegensatzes jener universellen Prinzipien. Dort steht vor allem die alte Klasse des Privatbesitzes, der Adel gemeinsam mit den Bauern dem Bourgeois der Stadt gegenüber. Dann treffen die beiden auf den „allgemeinen Stand“ [GPR § 205], „Beamtenschaft und Militärangehörige“.

b. Diese ständischen=klassenspezifischen Interessengegensätze werden durch die öffentliche Zusammenfassung in Form von Oberhaus, Unterhaus, den Organen der Regierung und den Ministerien übernommen. Der grundbesitzende Adel schickt seine Vertreter ins Oberhaus, Händler und Industrielle die ihren ins Unterhaus. Dadurch, dass letztere die Vorstände der zu ihnen gehörenden Korporationen ${ }^{69}$ in die Regierungsorgane entsenden, wird die Verbindung von bürgerlicher Gesellschaft und Staat gestärkt. Was die Beamten und Militärangehörigen betrifft, so „hat der allgemeine Stand [...] in seiner Bestimmung das Allgemeine für sich zum Zwecke seiner und zu seinem Boden" [GPR $\S$ 250: 273]. Demgegenüber hat der Stand der Grundbesitzenden ${ }^{70,71}$,an der Substantialität seines Familien- und Naturlebens in ihm selbst unmittelbar sein konkretes Allgemeines, in welchem er lebt [...]“" und ist der „substantielle Stand“ [GPR § 250: 273; GPR § 202, 203: 235]. Zwischen den beiden steht der Stand des Gewerbes. Dieser Stand „ist auf das Besondere wesentlich gerichtet und ihm ist daher vornehmlich die Korporation eigentümlich“ [Hegel 1981, GPR § 250: 273]. Seine Eigenschaft, das „[...]

68 Hier zitiert Hirata ohne Quellenangabe aus dem längeren Zusatz zu GPR § 182. Die Zusätze wurden von Hegels Schüler Eduard Gans (1797-1897) auf der Grundlage von Vorlesungen seines Lehrers verfasst und den GPR nachträglich hinzugefügt, vgl. Gans 1981 [1833]: 9.

69 Bei Hirata wörtlich „Berufsverbände“ (shokunō dantai 職能団体).

70 Bei Hegel steht an entsprechender Stelle: „Der ackerbauende Stand“ (GPR § 250: 273).

71 Tochi shoyūsha mibun 土地所有者身分. 
Besondere für sich“ zu sein, macht ihn zum „,reflektierten Stand“72 [Hegel 1979, § 523: 321, § 528: 322]. ${ }^{73}$

Die horizontale Verbindung zwischen den drei Schichten substanzieller, reflektierender und allgemeiner Stand verwirklicht sich in einer vertikalen Beziehung als Verhältnis von bürgerlicher Gesellschaft zum Staat. Sodann erhält die „Familie“, welche das natürliche Fundament der Sittlichkeit ist, in der bürgerlichen Gesellschaft als dem konkreten Ort ihres Lebens, [ihre] „Verfeinerung“. Im „politischen Staat“ als der Vereinigung von „fürstlicher Gewalt“, „Regierungsgewalt“ und „gesetzgebender Gewalt“ lässt sie sich selbst vollenden. Dies ist im Schaubild auf der folgenden Seite illustriert. ${ }^{74}$

c. Wie oben gezeigt, werden „Person“, „Eigentum“ und „Recht“, welche tatsächlich abstrakte Bestimmungen der Sittlichkeit sind, zu dem Zeitpunkt, in dem sich die Sittlichkeit im „Staat“ im weiteren Sinne verwirklicht, als Universelles ins Gegenteil gesetzt, und die „Moral“, welche die unmittelbare Form der Sittlichkeit ist, wird als das repräsentiert, was die Bildung in der bürgerlichen Gesellschaft vollendet. Dadurch wiederum wird im Gegenzug die Universalisierung des Bourgeois, der eine in den Sphären des „Rechts“ und der „Moral“ erzogene Privatperson (Privatmensch) ist, als „Mensch“ verwirklicht.

Entsprechend ist das Staatwerden bei Hegel die Sphäre des höchsten „Geistes“, in der Marxschen Dialektik des Umsturzes jenes Element, welches sich in Das Elend der Philosophie hin zu einer zukünftigen „Assoziation“ drehen kann.

d. Wenn Hegel solch eine große Bedeutung für Marx hat, wird deutlich, dass Marx' eigenes Denken nicht so eng ist, wie das häufig von ihm gezeichnete Bild des Wirtschaftswissenschaftlers. Die politisch-ökonomischen Kategorien [...]

72 In den Grundlinien heißt dieser auch „der reflektierende oder formelle“ Stand (GPR § 202: 235, Hervorhebung im Original).

73 Hier zitiert Hirata aus mehreren Textstellen und ohne Quellenverweis aus den Grundlinien der Philosophie des Rechts sowie aus der Enzyklopädie der philosophischen Wissenschaften im Grundriss. Die japanischen Zitate konnten wir den deutschen Entsprechungen zuordnen und somit nachträglich mit Quellenangaben in eckigen Klammern versehen. Zum Vergleich geben wir die betreffende Textstelle im Original wieder, vgl. Hirata 1989: 126: „官吏・軍人は「その使 命として普遍的なものを対自的におのが活動の目的とし、またおのれの基盤にする」。これ に対して、土地所有者身分は「おのれの家族生活と自然的生活とに実体化を、この生活その もののうちに直接おのれの具体的普遍者とすることによって、この普遍者のうちで生活す る」ところの、「実体的身分」である。この両者の間にあって商工業身分は「本来的に特殊 なものをめざし、その職能団体をおのれに固有のものとする」ところの身分であり、「その 特殊性において対自的なものである」ことをもって本性とする「反省的身分」である。“

74 Auf eine Reproduktion der Schaubilder an dieser Stelle wurde verzichtet. 
dieses großen Denkers, welche sich in der „Politikwissenschaft“ des jungen Marx und in der „Ökonomie“ seiner Zwischenphase erkennen lassen, sind in folgendem Schaubild, welches bereits in der Zeitschrift Shisō veröffentlicht wurde, dargestellt. Ich habe es überarbeitet und dabei den Fokus auf den Kontrast zu Hegel gelegt.

In diesem Schaubild sind die Momente, in denen die bürgerliche Gesellschaft dem Staat entfremdet wird, gleichzeitig die Vermittlungspunkte, welche den Staat in die bürgerliche Gesellschaft wiederaufnehmen. Dort können die ideologischen Apparate, die Hegemonieapparate des Systems sind, in Organe der Hegemonie umgewandelt werden, die das System aufheben.

e. Die Kategorien „politische Zivilisation“ und „Industrialismus“ innerhalb der oben abgebildeten Kategorien sind bis heute von der Marxologie kaum beachtet worden. Genauso verhält es sich mit den sich gegenüberstehenden Ausdrücken „Zentralismus“ und „Föderalismus“. Ferner ist es nicht nur in der „marxistischen Ökonomie“ sondern sogar auch in der „marxistischen Politikwissenschaft“ nie zu einer theoretischen und systematischen Erkenntnis der [Bedeutung] der „selbstverwalteten Kommunen“ gekommen. Auch ist nicht über die Unterscheidung von „Staatsbürger (citoyen)“ und „Volk“75 (peuple [pūpuru プープル]) sowie „Nation: ${ }^{76}$ (nation [nashion ナシオン])“ im Zusammenhang mit den ökonomischen Kategorien nachgedacht worden. Ist die universale Bedeutung der Machtzerstreuung und Dezentralisierung, die man im bourgeois-demokratischen Charakter der „Teilung der drei Gewalten“ erkennt, verstanden worden?

Dies sind die begrifflichen Apparate, welche mit der Unterscheidung von Staat und bürgerlicher Gesellschaft sowie von bürgerlicher Gesellschaft und bourgeoiser Gesellschaft in Zusammenhang stehen. Sie alle bieten einen ideellen Rahmen zur Erfassung der Wirklichkeit und sind es wert, heute noch einmal überdacht $\mathrm{zu}$ werden.

Die Gramsci-Forschung der Gegenwart weist berechtigter Weise darauf hin, dass Gramscis Hegemonietheorie bzw. [sein Konzept des] bürger-gesellschaftlichen Stellungskriegs ${ }^{77}$ an Hegels Theorie der bürgerlichen Gesellschaft angelehnt ist. Aber dieser wichtige Hinweis würde $\mathrm{zu}$ einer Nachlässigkeit

75 Jinmin人民.

76 Kokumin 国民.

77 Shimin shakaiteki zangōsenron 市民社会的塹壕戦論. 
ideengeschichtlicher Forschung, wenn man behauptete ,nicht Marx sondern Hegel' sei der ausschließliche Ursprung Gramscis Theorie der bürgerlichen Gesellschaft. Dies kann mit Hilfe des oben sehr einfach skizzierten Bildes von Marx verstanden werden. Wenn heute Hegel wieder aktuell ist, können wir auch Marx' reichen Begriffsapparat auf die Gegenwart anwenden. Ferner kann dadurch der wahre Wert Gramscis unser Gemeingut werden. ${ }^{78}$

\section{Bibliographie}

Althusser, Louis et al. (1996): Lire le Capital. Paris: Presses universitaires de France.

Avenell, Simon (2011): „Japan and the Global Revival of the 'Civil Society' Idea: Contemporaneity and the Retreat of Criticality“. Japan Forum 23.3: 311-338.

Bourgeois, Bernard (2005): „Der Begriff des Staates“. In: Grundlinien der Philosophie des Rechts. Edited by Georg W. F. Hegel. Hrsg. von Ludwig Siep. Berlin: Akademie Verlag, 217-242.

Buttigieg, Joseph A. (1991): „Gramscis Methode“. Das Argument 185: 9-26.

Dahrendorf, Ralf (1992): Der moderne soziale Konflikt: Essay zur Politik der Freiheit. Stuttgart: Deutsche Verlags-Anstalt.

Demirović, Alex (1991): „Zivilgesellschaft, Öffentlichkeit, Demokratie“. Das Argument 185: 41-55.

Deppe, Frank (2007): Krise und Erneuerung marxistischer Theorie: Anmerkungen eines Politikwissenschaftlers. http://linkesdsgruppe3.minuskel.de/fileadmin/linke.sds/MCH_ Reader/Frank_Deppe_-_KRISE_UND_ERNEUERUNG_MARXISTISCHER_THEORIE.pdf (15/02/ 2017).

Edwards, Bob / Diani, Mario / Foley, Michael W. (Hrsg.) (2001): Beyond Tocqueville: Civil Society and the Social Capital Debate in Comparative Perspective. Hanover: University Press of New England.

Edwards, Bob / Foley, Michael W. (1998): „Civil Society and Social Capital beyond Putnam“. American Behavioral Scientist 42.1: 124-139.

Foljanty-Jost, Gesine / Haufe, Karoline (2006): „Bürgerliche Gesellschaft versus Zivilgesellschaft: Die neuere Debatte in Japan“. Japanstudien 18: 247-269.

Gans, Eduard (1981 [1833]): „Vorrede“. In: Grundlinien der Philosophie des Rechts oder Naturrecht und Staatswissenschaft im Grundrisse. Edited by Georg W. F. Hegel. Berlin: Akademie-Verlag, 13-28.

Gramsci, Antonio / Bochmann, Klaus / Haug, Wolfgang F. (1991-2002): Gefängnishefte. Berlin: Argument-Verlag.

Gramsci, Antonio / Buttigieg, Joseph A. / Callari, Antonio (1992-2007): Prison Notebooks. New York: Columbia University Press.

78 Hier endet der bereits 1987 in Kikan Kuraishisu erschienene erste Teil des Aufsatzes. Der dort abgedruckte Schlusssatz ist in der Buchfassung von 1989 nicht mehr enthalten: „Diesen Aufsatz habe ich anlässlich der Teilnahmen an einem gemeinsamen Gedenken an Gramsci verfasst.“ 
Greffrath, Mathias (2016): Aktuelle Brisanz der Marxschen Kategorie. http://www.deutschland funk.de/re-das-kapital-1-6-aktuelle-brisanz-der-marxschen-kategorie.1184.de.html?dram: article_id=369501 (16/02/2017)

Habermas, Jürgen (1992): Faktizität und Geltung: Beiträge zur Diskurstheorie des Rechts und des demokratischen Rechtsstaats. Berlin: Suhrkamp.

Haug, Wolfgang F. (1988): „Gramsci und die Politik des Kulturellen“. Das Argument 167: 32-48. Haug, Wolfgang F. (1989): Gorbatschow: Versuch über den Zusammenhang seiner Gedanken. Hamburg: Argument-Verlag.

Haug, Wolfgang F. (1994): „Editorial“. Das Argument 206: 493-498.

Hegel, Georg W. F. (1979): „Enzyklopädie der philosophischen Wissenschaften im Grundrisse“. In: Werke 10. http://www.zeno.org/nid/20009179399 (01/09/2016).

Hegel, Georg W. F. (1981 [1833]): Grundlinien der Philosophie des Rechts oder Naturrecht und Staatswissenschaft im Grundrisse. Berlin: Akademie-Verlag.

Hettling, Manfred / Foljanty-Jost, Gesine (2009): „Formenwandel der Bürgergesellschaft: Japan und Deutschland im Vergleich“. Formenwandel der Bürgergesellschaft: Arbeitspapiere des Internationalen Graduiertenkollegs Halle Tōkyō 1: 1-63.

Hirata, Kiyoaki 平田清明 (1969): Shimin shakai to shakaishugi 市民社会と社会主. Tōkyō: Iwanami Shoten.

Hirata, Kiyoaki 平田清明 (1987a): „Marukusu shimin shakai gainen no saikō“マルクス市民社 会概念の再考. Kikan Kuraishisu 季刊クライシス 32: 62-69.

Hirata, Kiyoaki 平田清明 (1987b): „Gendai shihonshugi no seiji keizaigaku (joshō)“現代資本主 義の政治経済学 (序章). Shisō 思想 758: 46-69.

Hirata, Kiyoaki 平田清明 (1987c): „Gendai shihonshugi to shimin shakai“現代資本主義と市民 社会. In: Gendai shimin shakai no senkai 現代市民社会の旋回. Hrsg. von Hirata Kiyoaki / Yamada Toshio / Yagi Kiichirō 八木紀一郎. Kyōto: Shōwadō.

Hirata, Kiyoaki 平田清明 (1989): „Guramushi no shimin shakai gainen ni yosete“グラムシの市 民社会概念によせて. In: Ikite iru Guramushi: Botsugo gojū nen ki'nen bunshū 生きている グラムシ・没後 50 年記念論文集 [Lebender Gramsci: Schriftsammlung zum 50. Todestag]. Hrsg. von Ishidō Kiyotomo / lida Momo / Katagiri Kaoru 片桐薰. Tōkyō: Shakai Hyōronsha, 120-137.

Hirsch, Joachim (2002): „Tote Hunde wecken? Interview mit Joachim Hirsch zur Staatstheorie und Staatsableitung“. Arranca! 24, http://arranca.org/ausgabe/24/tote-hunde-wecken (15/02/2017).

Hishiyama, Izumi 菱山泉 (1995): „〈Tsuioku bun〉 Hirata Kiyoaki san o shinobu“〈追憶文〉平 田清明さんを偲ぶ. Keizai Ronsō 經濟論叢 156.6: 82-85.

Horstmann, Rolf-Peter (2005): „Hegels Theorie der bürgerlichen Gesellschaft“. In: Grundlinien der Philosophie des Rechts. Edited by Georg W. F. Hegel. Hrsg. von Ludwig Siep. Berlin: Akademie Verlag, 193-216.

Ishidō, Kiyotomo 石堂清倫 / lida, Momoいいだもも / Katagiri, Kaoru 片桐董 (Hrsg.) (1989): Ikite iru Guramushi: Botsugo gojū nen ki’nen bunshū 生きているグラムシ·没後 50 年記念 論文集. Tōkyō: Shakai Hyōronsha.

Kato, Tetsuro (1991): „Japanese Perception of the 1989 Eastern European Revolution“. Hitotsubashi Journal of Social Studies 23.1: 1-23.

Kessl, Fabian (2011): „Zivilgesellschaft“. In: Handbuch Sozialarbeit/Sozialpädagogik. Hrsg. von Hans-Uwe Otto / Hans Thiersch. München: Ernst Reinhardt, 1765-1774.

Lange, Joseph (1961): 100 Jahre Bürgergesellschaft Neuß 1861-1961. Neuß: Bürgergesellschaft. 
Liepitz, Alain (1992): „Vom Althusserismus zur ,Theorie der Regulation““. In: Hegemonie und Staat: Kapitalistische Regulation als Projekt und Prozess. Hrsg. von Alex Demirović / HansPeter Krebs / Thomas Sablowski. Münster: Westfälisches Dampfboot, 9-54.

Locke, John (1999): „Der Staat als Zusammenschluß zur Sicherung natürlicher Grundrechte“. In: Klassische Texte der Staatsphilosophie. Hrsg. von Norbert Hoerster. München: Deutscher Taschenbuch Verlag, 133-152.

Markner, Reinhard (1994): „Freiwillige Selbstbegrenzung: Jean L. Cohen und Andrew Aratos Theorie der bescheidenen Revolution“. Das Argument 206: 577-586.

Marx, Karl (1968 [1847]): Misère de la philosophie: Réponse à la philosophie de la misère de Proudhon. https://www.marxists.org/francais/marx/works/1847/06/km18470615.htm (01/09/2016).

Marx, Karl (1972 [1888]): „Das Elend der Philosophie“. Übers. von Eduard Bernstein und Karl Kautsky. In: Karl Marx /Friedrich Engels - Werke 4. Berlin: Dietz Verlag, http://www. mlwerke.de/me/me04/me04_063.htm (01/09/2016).

Marx, Karl (1976 [1844]): „Zur Judenfrage“. In: Karl Marx /Friedrich Engels - Werke 1. Berlin: Dietz Verlag, 347-377. http://www.mlwerke.de/me/me01/me01_347.htm (01/09/2016).

Pijl van der, Kees (1996): Vordenker der Weltpolitik. Opladen: Leske und Budrich.

Reiman, Michael (1987): Lenin, Stalin, Gorbačev: Kontinuität und Brüche in der sowjetischen Geschichte. Hamburg: Junius.

Seifert, Wolfgang (1999): „Notizen zum Begriffsverständnis von shimin shakai in Japan“. Japanstudien 11: 19-30.

Tsujinaka, Yutaka (2010): „Civil Society in Japan“. Inter Faculty 1. https://journal.hass.tsukuba. ac.jp/interfaculty/article/view/6/22 (01/09/2016).

Yagi, Kiichiro 八木紀一郎 (1995): „Eien ni gakumon no akari“永遠に学問の灯かがやけ. Keizai Ronsō 經濟論叢 156.6: 86-91.

Yagi, Kiichiro (2005): Problems of Japanese Economy and Public Space: Embedding Japanese Economy in the Civil Society. http://www.econ.kyoto-u.ac.jp/ yagi/yagi2005/linkfiles/ recent/Beijingyag.pdf (01/09/2016).

Załeski, Paweł (2007): „Propaganda społeczenstwo obywatelskiego w dobie transformacji“. Zeszyty Prasoznawcze 3/4: 41-51.

Zaleski, Pawel (2008): „Tocqueville on Civilian Society: A Romantic Vision of the Dichotomic Structure or Social Reality“. Archiv für Begriffsgeschichte 50: 260-266. 